\title{
TRIANGLES IN A COMPLETE CHROMATIC GRAPH
}

\author{
A. W. GOODMAN
}

(Received 26 January 1984)

Communicated by W. D. Wallis

\begin{abstract}
Suppose that in a complete graph on $N$ points, each edge is given arbitrarily either the color red or the color blue, but the total number of blue edges is fixed at $T$. We find the minimum number of monochromatic triangles in the graph as a function of $N$ and $T$. The maximum number of monochromatic triangles presents a more difficult problem. Here we propose a reasonable conjecture supported by examples.
\end{abstract}

1980 Mathematics subject classification (Amer. Math. Soc.): 05 C 15.

\section{Introduction}

Let $K_{N}$ be the complete graph with $N$ vertices (points) and suppose that each edge (line joining two vertices) is assigned exactly one of two possible colors, here always blue or red. After the coloring let $B$ be the number of monochromatic (solid) blue triangles, and let $R$ be the number of solid red triangles. The minimum of $B+R$ for each $N>0$ was determined by the author [2]. Later Sauvé [4] gave a simpler proof of the same theorem using an ingenious method of assigning weights to each pair of edges at each vertex. The maximum of $B+R$ is trivially $N(N-1)(N-2) / 6$, and the associated extremal graph is obtained by painting every edge blue, or painting every edge red.

Suppose that we modify the problem by considering only those colorings of $K_{N}$ in which the number of blue edges is $T$ where $T$ is a fixed preassigned number. This also fixes the number of red edges $U$, and of course $U=N(N-1) / 2-T$.

(c) 1985 Australian Mathematical Society $0263-6115 / 85 \$ A 2.00+0.00$ 
Let $m(N, T)$ be the minimum of $B+R$ for all colorings with $T$ blue edges, and let $M(N, T)$ be the maximum of $B+R$ under the same conditions. In this work we find $m(N, T)$ explicitly for every pair of integers $N, T$, with $0 \leqslant T \leqslant$ $N(N-1) / 2$ and $0 \leqslant N<\infty$.

The problem of finding $M(N, T)$ seems to be more difficult. In Section 4 we discuss the difficulties and we propose a reasonable conjecture for the form of the solution.

\section{The basic equations}

Following Sauvé [4], we attach the weight 2 to each pair of blue lines issuing from a vertex of the graph. We assign the same weight 2 , to each pair of red lines. For a mixed pair, one edge blue and one edge red we assign the weight -1 . Let $b_{j}$ and $r_{j}$ be the number of blue edges and red edges respectively at the point $P_{j}, j=1,2, \ldots, N$. Then for each $j$

$$
b_{j}+r_{j}=N-1 \text {, }
$$

and

$$
\sum b_{j}=2 T, \quad \sum r_{j}=2 U,
$$

where all sums run from 1 to $N$ unless otherwise noted. When we compute the weight of the edges meeting at $\boldsymbol{P}_{j}$ in accordance with the above assignment of weights, we obtain

$$
b_{j}\left(b_{j}-1\right)+r_{j}\left(r_{j}-1\right)-b_{j} r_{j} .
$$

Then the total weight of the colored graph is

$$
W=\sum b_{j}\left(b_{j}-1\right)+\sum r_{j}\left(r_{j}-1\right)-\sum b_{j} r_{j} .
$$

On the other hand if we examine the weight of each triangle, the weight of a monochromatic triangle is 6 , and the weight of a mixed triangle is $2-1-1=0$. Then for the total weight we also have $B+R=W / 6$. Hence for any coloring,

$$
B+R=\frac{1}{6} \sum\left[b_{j}\left(b_{j}-1\right)+r_{j}\left(r_{j}-1\right)-b_{j} r_{j}\right] .
$$

Now $b(b-1)+r(r-1)-b r=(b+r)^{2}-(b+r)-3 b r$. If we use (2.1) and (2.2), then the formula (2.4) for $B+R$ gives

$$
\begin{aligned}
B+R & =\frac{1}{6} \sum\left[\left(b_{j}+r_{j}\right)^{2}-\left(b_{j}+r_{j}\right)-3 b_{j}\left(N-1-b_{j}\right)\right] \\
& =\frac{1}{6}\left[N(N-1)^{2}-N(N-1)-6 T(N-1)+3 \sum b_{j}^{2}\right],
\end{aligned}
$$


or

$$
B+R=\frac{N(N-1)(N-2)}{6}-T(N-1)+\frac{1}{2} \sum b_{j}^{2} .
$$

Thus to minimize or maximize $B+R$ with $T$ constant it is sufficient to minimize or maximize $Q \equiv \sum b_{j}^{2}$.

We observe that the red edges have disappeared in (2.5). Hence the problem of minimizing or maximizing $B+R$ is equivalent to the problem of finding extreme values of $Q$ for an incomplete graph on $N$ vertices with $T$ edges. With this interpretation, $R$ is the number of independent triples; that is, the number of sets of three vertices with no edges joining any pair.

A sequence of integers $\left(b_{1}, b_{2}, \ldots, b_{N}\right)$ with

$$
N-1 \geqslant b_{1} \geqslant b_{2} \geqslant \cdots \geqslant b_{N} \geqslant 0,
$$

is called a graph sequence (degree sequence) if there is a graph on $N$ vertices such that the vertex $P_{j}$ has degree $b_{j}$. Since we can always number the vertices in such a way that (2.6) is satisfied, there is no loss of generality in assuming that the sequence $\left(b_{1}, b_{2}, \ldots, b_{N}\right)$ is nonincreasing. A sequence can satisfy (2.6) and still not be a graph sequence. For example $(3,2,1,0)$ is not the sequence of degrees for any graph on 4 vertices. A necessary and sufficient condition for a sequence to be a graph sequence can be found in the text by Behzad and Chartrand [1, p. 12]. For our objective we will need the following necessary conditions:

$$
\sum b_{j}=2 T, \text { an even number; }
$$

$$
b_{1} \leqslant \text { the number of nonzero } b_{j} \text { in }\left(b_{2}, b_{3}, \ldots, b_{N}\right) \text {, }
$$

and for each $k$ with $0<k \leqslant N$

$$
\sum_{j=1}^{k} b_{j}-\sum_{j=k+1}^{N} b_{j} \leqslant k(k-1) .
$$

\section{The minimum number of monochromatic triangles}

Suppose that in a sequence (2.6) there are two indices $j$ and $k$ for which $b_{j} \geqslant b_{k}+2$. We replace $b_{j}$ by $b_{j}-1$ and $b_{k}$ by $b_{k}+1$. Since

$$
b_{j}^{2}+b_{k}^{2}>\left(b_{j}-1\right)^{2}+\left(b_{k}+1\right)^{2} \text {, }
$$

this replacement will decrease $Q \equiv \sum b_{j}^{2}$. Thus

LEMMA 1. The minimum of $Q$ over all sequences of integers (2.6) that satisfy (2.7) occurs for a sequence in which either all $b_{j}$ are the same, or no two differ by more than 1. 
To compute this minimum, we set

$$
\frac{2 T}{N}=q+\frac{\rho}{N}, \quad 0 \leqslant \rho<N,
$$

where $q$ and $\rho$ are integers. Then the minimizing sequence has the form

$$
S=(q+1, q+1, \ldots, q+1, q, q, \ldots, q),
$$

where there are $\rho$ terms $b_{j}=q+1$, and the remaining $b_{j}=q$. A brief computation with this sequence and (2.5) gives

$$
B+R \geqslant \frac{N(N-1)(N-2)}{6}-T(N-q-1)+\frac{\rho(q+1)}{2} .
$$

To show that this lower bound is sharp we must produce a graph that has the required sequence as a graph sequence. From (3.1) we see that $2 T=N q+\rho$ must be even. Thus certain combinations of $N, q$ and $\rho$ cannot occur. Of the eight possibilities (with respect to parity) we can not have (1) $N$ odd, $q$ odd, $\rho$ even, (2) $N$ odd, $q$ even, $\rho$ odd, (3) $N$ even, $q$ odd, $\rho$ odd, and (4) $N$ even, $q$ even, $\rho$ odd.

Suppose that $q$ is even. We may visualize the vertices arranged in order on a circle. Join each vertex with its nearest $q / 2$ neighbors on either side by blue edges. Thus $P_{j} P_{k}$ is blue if $|j-k| \leqslant q / 2$, where $j$ or $k$ are reduced $\bmod N$ if necessary. This gives each $b_{j}=q$. Now color $P_{j} P_{k}$ blue for $k=j+1+q / 2$ and $j=$ $1,2, \ldots, \rho$. Then this graph has the sequence (3.2) with respect to blue edges, and $\sum b_{j}=2 T$.

Suppose that $q$ is odd. Then $q+1$ is even and we proceed as in the first case joining each vertex by a blue edge to its nearest $(q+1) / 2$ neighbors on either side. Thus $P_{j} P_{k}$ is blue if $|j-k| \leqslant(q+1) / 2$. This gives too many blue edges, so we change some to red as follows. If $N$ is even, then $\rho$ is even, and $N-\rho$ is even. Then change from blue to red the edges $P_{N} P_{N-1}, P_{N-2} P_{N-3}, \ldots$ until $(N-\rho) / 2$ edges have been changed.

If $N$ is odd, and $q$ is odd, then $\rho$ is odd and $N-\rho$ is even. We proceed as before to change from blue to red $P_{N} P_{N-1}, P_{N-2} P_{N-3}, \ldots$ until $(N-\rho) / 2$ edges have been changed. Thus in every possible case we have a graph with the sequence (3.2) with respect to blue edges. This completes the proof of

THEOREM 1. Suppose that the edges of a complete graph on $N$ vertices are colored with two colors, red and blue, coloring $T$ edges blue, and the remaining red. Then minimum $(R+B)$ is given by the right side of (3.3), where $\rho$ and $q$ are defined by (3.1). 


\section{The maximum number of monochromatic triangles}

The same computation that gave Lemma 1 shows that for a maximum we should make the early $b_{j}$ as large as possible. Suppose that the sequence is subject only to the conditions (2.6) and (2.7). Define integers $q$ and $\rho$ by $2 T=q(N-1)$ $+\rho$ where $q \geqslant 0$ and $0 \leqslant \rho<N-1$. Then the sequence $S=(N-1, \ldots, N-$ $1, \rho, 0, \ldots, 0)$ with $N-1$ repeated $q$ times will make $\Sigma b_{j}^{2}$ a maximum. But $S$ is not a graph sequence except in the trivial cases, all $b_{j}=0$ or all $b_{j}=N-1$.

To find graph sequences with large $\Sigma b_{j}^{2}$, we introduce two special graphs.

First, there are nonnegative integers $q$ and $\rho$ such that

$$
T=q(N-1)-\frac{q(q-1)}{2}+\rho, \quad 0 \leqslant \rho \leqslant N-q-2 .
$$

With these integers let $G^{*}$ be the graph obtained as follows. Let $P_{1}, P_{2}, \ldots, P_{q}$ be a complete subgraph $K_{q}$ on $q$ vertices. Join each vertex of $K^{q}$ with each of the remaining vertices $P_{q+1}, \ldots, P_{N}$. Finally join $P_{q+1}$ to $P_{q+2}, \ldots, P_{q+\rho+1}$ using $\rho$ edges. Of course this set of vertices is empty if $\rho=0$. Then the graph sequence for this graph is

$$
S^{*}=(N-1, \ldots, N-1, q+\rho, q+1, \ldots, q+1, q, \ldots, q),
$$

where $N-1$ occurs $q$ times, $q+1$ occurs $\rho$ times, and $q$ occurs $N-q-\rho-1$ times. An easy computation shows that $\Sigma b_{j}=2 T$ for $S^{*}$. Since $S^{*}$ is the sequence for $G^{*}$, it must satisfy the conditions (2.7), (2.8), and (2.9). Further when $k=q+1$, the sequence $S^{*}$ gives equality in (2.9). Thus the degrees, $b_{1}, b_{2}, \ldots, b_{q+1}$ are as large as possible. This suggests that $G^{*}$ makes $B+R$ a maximum, when the edges of $G^{*}$ are colored blue and the remaining edges of $K_{N}$ are colored red. We will see shortly that this is not always the case.

Next we define non negative integers $v$ and $\lambda$ by

$$
\frac{2 T}{v(v-1)}=1+\frac{2 \lambda}{v(v-1)}, \quad 0 \leqslant \lambda<v .
$$

Let $G_{v}$ be the graph defined as follows. The vertices $P_{1}, P_{2}, \ldots, P_{v}$ form a complete subgraph on $v$ vertices. Then join each of $P_{1}, P_{2}, \ldots, P_{\lambda}$ with $P_{v+1}$ by an edge. The graph sequence for $G_{v}$ is

$$
S_{v}=(v, \ldots, v, v-1, \ldots, v-1, \lambda, 0, \ldots, 0)
$$

where $v$ occurs $\lambda$ times and $v-1$ occurs $v-\lambda$ times. An easy computation shows that $\Sigma b_{j}=2 T$ for $S_{v}$. As we will see, when the edges of $G_{v}$ are colored blue and the remaining edges of $K_{N}$ are colored red, this graph occasionally gives a maximum for $R+B$ when $G^{*}$ fails.

We now look at several examples. First suppose that $N=4$ and $T=3$. Then

$$
S^{*}=(3,1,1,1), \quad \sum b_{j}^{2}=12,
$$


and

$$
S_{v}=(2,2,2,0), \quad \sum b_{j}^{2}=12 .
$$

Therefore both graphs give the same maximum. In fact $S^{*}$ is the complement of $S_{v}$ so $R+B$ must be the same for both graphs.

Next, let $N=5$ and $T=4$. Then

$$
\begin{array}{ll}
S^{*}=(4,1,1,1,1), & \sum b_{j}^{2}=20, \\
S_{v}=(2,2,2,2,0), & \sum b_{j}^{2}=16 .
\end{array}
$$

This time $S^{*}$ provides the maximum for $R+B$.

Finally let $N=7$ and $T=10$. Then

$$
\begin{array}{ll}
S^{*}=(6,5,2,2,2,2,1), & \sum b_{j}^{2}=78, \\
S_{v}=(4,4,4,4,4,0,0), & \sum b_{j}^{2}=80 .
\end{array}
$$

This time it is $S_{v}$ that gives a maximum.

For the sequence $S^{*}$ in (4.2), we find

$$
\sum b_{j}^{2}=q(N-1)^{2}+(4 q+\rho+1) \rho+q^{2}(N-q),
$$

an expression which does not seem to simplify.

For $S_{v}$ in (4.4) we have

$$
\sum b_{j}^{2}=\lambda v^{2}+(v-\lambda)(v-1)^{2}+\lambda^{2}=2 T(v-1)+\lambda(\lambda-1) .
$$

CONJECTURE. If a complete graph on $N$ vertices is colored with $T$ blue edges and the rest red edges, then $\max (B+R)$ occurs either for the graph $G^{*}$ or $G_{v}$. If this is the case, then $\max (B+R)$ is given by (2.5) where either (1) $q$ and $\rho$ are defined by (4.1) and $\Sigma b_{j}^{2}$ is given by (4.5), or (2) $v$ and $\lambda$ are defined by (4.3) and $\sum b_{j}^{2}$ is given by (4.6).

There is a relation between $S^{*}$ and $S_{v}$ which we now develop. First observe that the integers $N, T, q$, and $\rho$ determine $S^{*}$ uniquely and conversely $S^{*}$ determines $N, T, q$, and $\rho$ uniquely. Similarly there is a one-to-one correspondence between the integers $N, T, v$, and $\lambda$ and the graph sequences $S_{v}$. Now consider $\tilde{G}$ the complement of $G$. If $G$ is an incomplete graph then $\tilde{G}$ has an edge $P_{i} P_{j}$ if and only if $P_{i} P_{j}$ is not an edge of $G$. Or if $G$ is regarded as a complete graph with two colors, then the colors are interchanged in $\tilde{G}$. Let $\tilde{S}^{*}$ be the graph sequence obtained from $S^{*}$ when we replace $b_{j}$ by $N-1-b_{j}$ for $j=1,2, \ldots, N$ and then reverse the order. With this notation we have

THEOREM 2. The sequence $\tilde{S}^{*}$ is the graph sequence $S_{v}$ for the graph $\tilde{G}^{*}$. 
Proof. If $S^{*}$ is the sequence (4.2) then

$$
\begin{aligned}
& \tilde{S}^{*}=(N-1-q, \ldots, N-1-q, N-2-q, \ldots, \\
&N-2-q, N-1-q-\rho, 0, \ldots, 0),
\end{aligned}
$$

where $N-1-q$ occurs $N-q-\rho-1$ times, $N-2-q$ occurs $\rho$ times and 0 occurs $q$ times. We set $v=N-1-q$ and $\lambda=N-q-\rho-1$. Then

$$
\tilde{S}^{*}=(v, \ldots, v, v-1, \ldots, v-1, \lambda, 0, \ldots, 0),
$$

where $v$ occurs $v-\rho=\lambda$ times, $v-1$ occurs $\rho=v-\lambda$ times and 0 occurs $q=N-v-1$ times, (see equation (4.4)). Then $\tilde{S}^{*}$ is the sequence $S_{v}$ for $\tilde{G}^{*}$.

CoRollary. If $G^{*}$ gives the maximum $R+B$ for all two-color graphs on $N$ points with $T$ blue edges, then $G_{v}$ gives the maximum $R+B$ for all two-color graphs on $N$ points with $U=N(N-1) / 2-T$ blue edges.

\section{Bounds for other combinations}

In a coloring of $K_{N}$ with blue and red, let $B B R$ be the number of triangles with two blue edges and one red edge. Similarly let $B R R$ be the number of triangles with one blue edge and two red edges.

Let us attach the weight $x$ to each pair of blue edges, the weight $y$ to each mixed pair, and the weight $z$ to each pair of red edges. Again we compute the weight of $K_{N}$ in two different ways. Following the notation and method of section 2 , with this more general system of weights, we obtain

$$
\begin{aligned}
3 x B & +(x+2 y) B B R+(z+2 y) B R R+3 z R \\
& =\sum\left[\frac{x}{2} b_{j}\left(b_{j}-1\right)+\frac{z}{2} r_{j}\left(r_{j}-1\right)+y b_{j} r_{j}\right] .
\end{aligned}
$$

We replace $r_{j}$ by $N-1-b_{j}$ and use $\Sigma b_{j}+2 T$. Then (5.1) becomes

$$
\begin{aligned}
3 x B+(x+2 y) & B B R+(z+2 y) B R R+3 z R \\
= & \frac{z}{2} N(N-1)(N-2)+[2 y(N-1)-x-z(2 N-3)] T \\
& +\left(\frac{x+z}{2}-y\right) \sum b_{j}^{2} .
\end{aligned}
$$

Various selections of weights $(x, y, z)$ in (5.2) give special relations. The selection $(2,-1,2)$ gives equation $(2.5)$. The selection $(0,1,0)$ gives

$$
2(B B R+B R R)=2(N-1) T-\sum b_{j}^{2} .
$$

Equation (5.3) also follows from (2.5) and the fact that the total number of triangles is $N(N-1)(N-2) / 6$, (try $(x, y, z)=(1,1,1))$.

Let $(x, y, z)=(1,0,0)$. Then (5.2) yields

$$
3 B+B B R=-T+\frac{1}{2} \sum b_{j}^{2} .
$$


Further $(x, y, z)=(2,-1,0)$ gives

$$
3 B-B R R=-N T+\sum b_{j}^{2} .
$$

Finally $(x, y, z)=(0,1,1)$ gives

$$
2 B B R+3 B R R+3 R=\frac{1}{2} N(N-1)(N-2)+T-\frac{1}{2} \sum b_{j}^{2} .
$$

These equations are not independent. Since the vectors $(1,1,1),(0,1,0)$, and $(1,0,0)$ form a basis for $E^{3}$, it follows that any equation that can be derived from (5.2) can also be obtained from (5.3), (5.4), and the fact that the total number of triangles is $\left(\begin{array}{c}N \\ 3\end{array}\right)$.

The methods that gave Theorem 1 , also yield

THEOREM 3. Suppose that the edges of $K_{N}$ are colored with two colors red and blue and that $T$ edges are colored blue. Define $\rho$ and $q$ by equation (3.1). Then we have the following sharp bounds:

$$
\begin{gathered}
B B R+B R R \leqslant T(N-q-1)-\frac{\rho(q+1)}{2} ; \\
3 B+B B R \geqslant T(q-1)+\frac{\rho(q+1)}{2} ; \\
3 B-B R R \geqslant T(2 q-N)+\rho(q+1) ;
\end{gathered}
$$

and

(5.10) $2 B B R+3 B R R+3 R \leqslant \frac{1}{2} N(N-1)(N-2)-T(q-1)-\frac{\rho(q+1)}{2}$.

Of course (5.7) follows directly from the bound (3.3).

\section{References}

[1] Mehdi Behzad and Gary Chartrand, Introduction to the theory of graphs (Allyn and Bacon, Inc., Boston, 1971).

[2] A. W. Goodman, 'On sets of acquaintances and strangers at any party', Amer. Math. Monthly' 66 (1959), 778-783.

[3] A. W. Goodman, 'Triangles in a complete chromatic graph with three colors', Discrete Math., to appear.

[4] Leopold Sauvé, 'On chromatic graphs', Amer. Math. Monthly 68 (1961), 107-111.

Department of Mathematics

University of South Florida

Tampa, Florida 33620

U.S.A. 